\title{
Ambient vibration testing and operational modal analysis of monopole telecoms structures
}

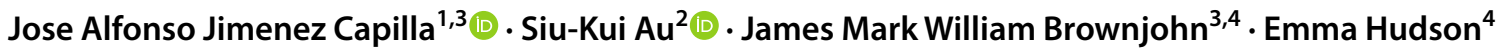

Received: 21 August 2020 / Revised: 19 May 2021 / Accepted: 28 May 2021 / Published online: 12 June 2021

(c) The Author(s) 2021

\begin{abstract}
A structural health monitoring (SHM) system was developed to study the ambient response of monopole communication structures in the UK operated by Arqiva Ltd. The exercise had several purposes that included the evaluation of the SHM system itself and the system identification procedures applied to the data, followed by analysis of the evaluated modal properties to validate the current analytical models, structural assessments and standardised design procedures advising on dynamics actions. This paper describes the instrumentation and procedures used during monitoring of a lightweight flexible $14.5 \mathrm{~m}$ tubular tapered monopole supporting an array of mobile telecoms antennas. A Bayesian OMA (BAYOMA) approach is implemented to identify structural modal properties under different time windows as comparison for further assessments. Results from stochastic subspace identification are also obtained and compared. The correlation between modal properties and monitoring wind-response data reveals specific tendencies such as nonlinear stiffness behaviour, the existence of aerodynamic damping and typical directionality of the mode shapes with future implications for reformulation of current methods of assessing dynamics on monopole.
\end{abstract}

Keywords Monopoles $\cdot$ Telecoms structures $\cdot$ Monitoring system $\cdot$ Operational modal analysis

\section{Introduction}

Due to requirements for new fifth generation of mobile telecoms (5G), structures for mounting antenna equipment at height need to be optimised to sustain the increased loads. The weakest structures in this respect are 'monopoles', i.e., slender steel cylindrical structures which reach from 10 to

Jose Alfonso Jimenez Capilla

jj379@exeter.ac.uk; jose.alfonso.jc@gmail.com

Siu-Kui Au

ivanau@ntu.edu.sg; siukuiau@gmail.com

James Mark William Brownjohn

J.Brownjohn@exeter.ac.uk

Emma Hudson

e.j.hudson@fullscaledynamics.com

1 Arqiva Ltd, Winchester, UK

2 School of Civil and Environmental Engineering, Nanyang Technological University, 50 Nanyang Avenue, Singapore S639678, Singapore

3 Structural Dynamics, University of Exeter, Exeter, UK

4 Full Scale Dynamics Ltd, Sheffield, UK
$30 \mathrm{~m}$ above ground to place equipment at elevated positions for highest possible coverage. The introduction of new technology such as $5 \mathrm{G}$ is presently requiring new heavier and larger equipment payloads that change monopole dynamic behaviour under wind loading.

Structural engineers, following recommendations written in national standards, assess main modal parameters (MPs) to account for dynamic effects via typical quasi-static wind-loading analysis. The reliability of MPs used in these approaches have been in doubt by consultants during the last decade. Further investigations based on newly acquired fullscale performance data and structural identification methods are required.

With respect to damping, the design codes [1-3] consider the ways a monopole structure absorbs energy during vibration by different means, i.e., externally provided by dampers, aerodynamically due to the wind itself, through structural damping arising from distortion of the materials and friction at connections and finally through interaction of foundations radiating energy into the soils. Effects related to e.g., geometry of the structure, location of the site, soil conditions on site or hold equipment, define dynamic parameters that are 
important during design and review of capabilities during the life of the structure [4].

Currently, a high percentage of monopole structures are invalidated to support new antennas according to standards, i.e., the most modern Eurocode BS EN 1991-1-4: 2005 "Wind Actions" [1], British Standard BS 8100: Part 1:1986 [2] and the Institution of Lighting Engineers (UK) Technical Report Number 7 [3]. This lack of capacity means extra cost for owners and customers who need to agree expensive structural strengthening or tower replacements. This would also involve delay in the total implementation of $5 \mathrm{G}$ programmes with additional costs for the end users.

To avoid such delays and extra costs, there is need for further development to improve the current approaches regarding dynamic performance in which the values of structural damping were proved to be very conservative for a high percentage of the structures. Predicting natural frequencies with numerical modal analyses with precise agreement with in-situ values (e.g., less than 10\%) is found to be challenging. This can be attributed to, e.g., lack of knowledge and variability in the effectiveness of foundation-soil to dissipate energy and problems with the aerodynamic-damping formulation using inappropriate drag coefficients working under turbulent wind loading.

There are also concerns around fatigue in ground level connections with a number of failures identified during the previous two decades. Some of these concerns are best addressed by conventional inspection of the inner connection of the base flange, but a better knowledge of dynamics and a continuous record of their evolution can provide a wealth of information capable of addressing many of the key concerns. This impulse comes from structure owners that are required to add new antenna payloads to structures already at capacity according to the current standards that prescribe the obsolete damping values. Several investigations are setting a new perspective in this issue.

To obtain in-service performance data, a Structural Health Monitoring (SHM) system was developed that aims at acquiring information about wind loading and response of the structure under a variety of working conditions. There have been relatively few SHM systems installed in telecoms towers, so far none (to authors' knowledge) in monopoles. Historically monitoring systems aimed to study wind environment [5] and/or strain (for fatigue purposes) mostly in guyed towers, e.g., a trio of British high guyed masts [6] with a few exercises focussed on acceleration data $[7,8]$ including a recent study on a high guyed mast studying the effectiveness of a tuned liquid damper in controlling effects of vortex shedding [9].

This study used a relatively simple SHM system for detailed non-destructive evaluation of the dynamics of the structure under several loading conditions, to remodel structural assessments and to evaluate fatigue life and (if required) provide information to design a retrofitted damper. Signals from a mechanical anemometer and two uniaxial horizontal accelerometers were collected using a ground level data logger. The system has been sequentially deployed in several structures but this paper will cover the data obtained during more than 2 months on a Portasilo Monopole in St Ives; see Fig. 1.

This type of monitoring systems will be more feasible in future. New acquisition methodologies [9] based on economically viable equipment coupled with data delivery and analysis would better support decisions on structural management. For this purpose and to improve current approaches, the present paper introduces modal identification procedures to identify and explain dynamic load/response mechanisms.

This exercise executes the operational modal analysis (OMA) procedure based on innovative Fast Bayesian
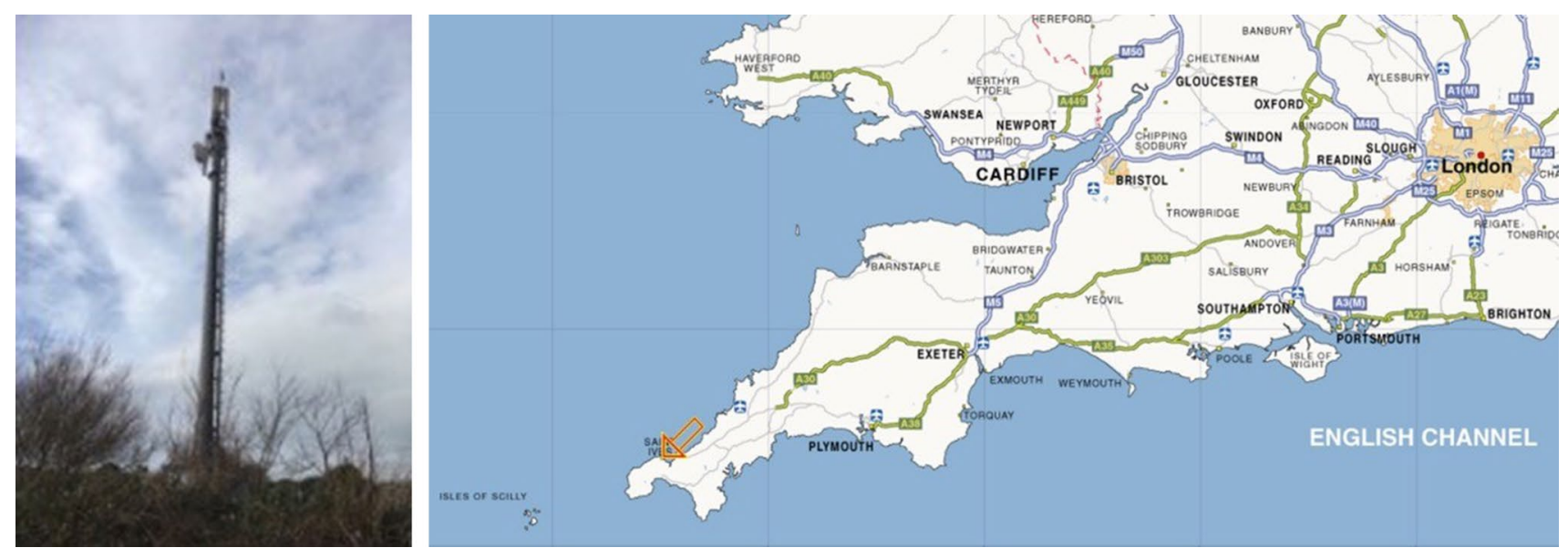

Fig. 1 (Left) Picture of St Ives FC Portasilo monopole. SHM system install. Arqiva. (Right) Location of St Ives in South-West of the UK 
ambient modal identification method (BAYOMA) [10, 11], which has been applied to other civil structural types such as buildings, bridges and lighthouses [12]. This paper compares and contrasts the procedures applied to assessing monopole performance using three different lengths of data time frame $\left(T_{\mathrm{fr}}\right)$. It will set a final decision where to base technical conclusions in terms of dynamic parameters like frequencies, damping or mode shapes to apply to other SHM.

Results are also compared with those from Stochastic Subspace Identification method (SSI, [13]), which is one conventional method used in several types of dynamic sensitive structures [14] for the last two decades, here implemented by ARTeMIS software [15]. In the case of the monopole studies, BAYOMA applied to $T_{\mathrm{fr}}=10 \mathrm{~min}$ time frames is shown to be advantageous because it provides means and variances of all MPs, including mode shapes and the power spectral density matrix of modal forces along the whole range of amplitudes found during the monitoring. This is the background to a project to install SHM system for structural diagnosis of several types of telecom structures in the existing portfolio.

\section{Structural health monitoring system in short telecoms structures}

As stated, the main purpose of this SHM exercise was to obtain information on structure response under different wind load cases. Due to the geometry and the slenderness of the structure, it can behave under two different scenarios [16]; along-wind buffeting where response depends on the wind intensity, and vortex shedding at relatively low wind speed driving strong cross-wind response when shedding frequency matches a natural frequency. To define both effects requires knowledge of the wind from the anemometer, and of the structure dynamics (main frequencies, structural and aerodynamic damping and mode shapes) from the accelerometers.

The implemented SHM system (Fig. 2) had the following components:

- To capture the monopole response the system is based on two monoaxial PCB accelerometers model 393B04, with a sensitivity of $1000 \mathrm{mV} / \mathrm{g}$ able to measure between $\pm 5 \mathrm{~g}\left( \pm 49 \mathrm{~m} / \mathrm{s}^{2} \mathrm{pk}\right)$ placed in a waterproof box.

- A RM Young mechanical anemometer to capture wind loading in terms of horizontal wind speed and direction
Fig. 2 SHM system overview. (Left) Elevation sketch of SHM. (Upper Right) Response Box containing two Mono-Axial Accelerometers. (Centre Right) Anemometer located on site in St Ives. (Downer Right) Logger box

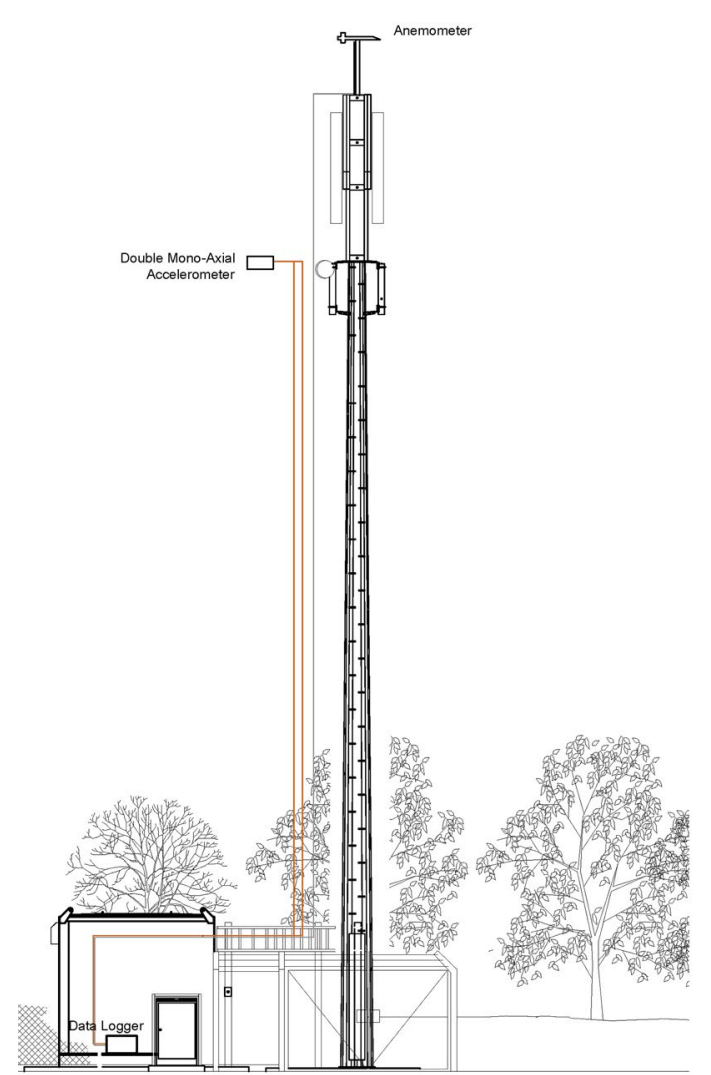

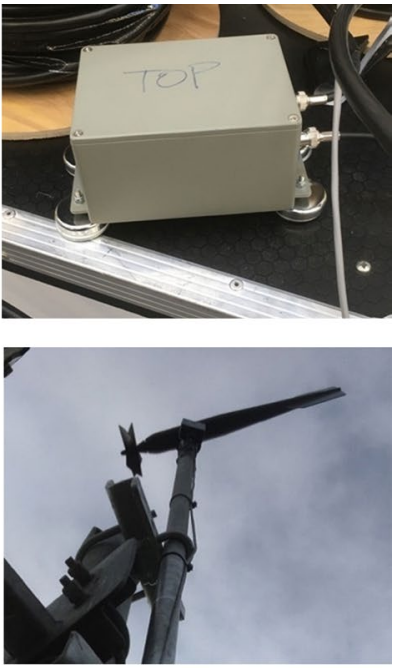

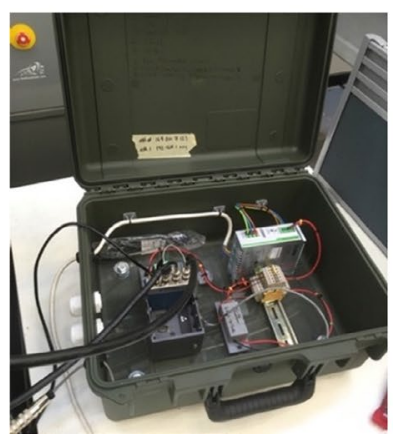


(with respect to North). This produces an AC sine wave signal with frequency proportional to wind speed with three cycles per propeller revolution $(0.0980 \mathrm{~m} / \mathrm{s}$ per $\mathrm{Hz}$ ) and FFT of 2-s data blocks was used to extract wind speed values up to $100 \mathrm{~m} / \mathrm{s}$ with operating temperature between -50 and $+50{ }^{\circ} \mathrm{C}$, far beyond the expected range in this application. A threshold minimum frequency corresponding to a wind speed of $2 \mathrm{~m} / \mathrm{s}$ is set to avoid erroneous readings for low wind speeds.

- National Instruments (NI) cRIO-9064 with NI USB-9234 input module installed in a protected chassis for data logging. The system oversamples at $5.120 \mathrm{kHz}$ to capture the anemometer signal. Each block of data acquired was resampled to $128 \mathrm{~Hz}$ with FIR filter to avoid aliasing, with $120 \mathrm{~dB}$ alias rejection and normalised bandwidth of 0.4536 .

- Cables run from sensors to logger and requiring proper protection due to extreme outdoor environmental conditions.

- Steel mounting designed to place the anemometer beyond the reach of radio frequency interference due to the antennae installed below. Likewise, attachments were designed to place the accelerometer at highest horizontal level available in the structure, around at $15 \mathrm{~m}$, as shown in Fig. 2.

\section{SHM installation in St Ives FC monopole}

\subsection{Monopole structural details}

Meeting a requirement for the most effective deployment of the SHM system, the first suggested site in the Arqiva portfolio was a short Portasilo monopole of $14.5 \mathrm{~m}$ located at St Ives Football Club, Fig. 1, in south west UK. This type of monopole is the most usual type in the Arqiva portfolio, being lightweight and flexible. Monopoles of this type were found to have the highest dynamic amplification factors [3] during structural assessments, limiting their capacity for future changes. They have been found to be prone to failure at base level due to fatigue over short lifespans (less than 10 years). St Ives is located in Cornwall, a region with the highest designing basic windspeed in the UK, 23-24 m/s. In addition to that, the site is less than $1 \mathrm{~km}$ from the sea shore, with theoretical high wind speeds and relatively low turbulence with terrain categories I and II. In terms of topography, the site is placed at the top of hill surrounded by residential houses to the north and east and clear fields to the south and west. Consequently, significant orographic influence is expected from those wind directions.

Arqiva provides a complete knowledge of geometric and mechanical characteristics of the mass and stiffness of the structure and substructure (foundations), masses of existing antennas given by manufacturer, and soils investigations carried out after geotechnical field tests. The structure is a monopole comprising S275J0 steel for main core and plates of $25 \mathrm{~mm}$ thick S355J2. There are two sections: a tubular tapered panel between ground level and $14.3 \mathrm{~m}$ and a triangular steelwork headframe suitable to accommodate mobile antennas. Also, there is an external spine ladder with fall arrest system. For loading, three antenna panels are installed at the upper part, with a small plate antenna at $14.3 \mathrm{~m}$, with all necessary cables run internally. This structure is joined to the foundation thorough a flange plate and 12 Class $8.8 \mathrm{M} 24$ bolts, without grouting. The foundation is a concrete block of dimensions $3.6 \times 3.6 \times 0.85 \mathrm{~m}$ as Table 1 . The chosen site was proposed for a decommission and replacement under the implementation of $5 \mathrm{G}$ (Fig. 3).

The equipment was installed on the morning of 22nd of November 2018 and retrieved on 5th of February 2019 by two engineers and two expert climbers, fixing the anemometer with necessary steelwork at $17.5 \mathrm{~m}$ and the accelerometers at $14.5 \mathrm{~m}$. No further climbing was allowed to avoid inclusion of non-ambient loading in the monitoring period.

\subsection{Full-scale wind loading and response}

The 76 days of continuous measurements over the length of the monitoring were divided into consecutive data files of 10-min time frame. Each file comprises five channels: acceleration records of both accelerometers, wind speed, wind direction and temperature.

The data extracted were plotted along a time line for the whole monitoring, extracting mean values and gust factors for wind analysis. The time line of wind speed appears in Fig. 4 showing some high wind speeds, exceeding $40 \mathrm{~m} / \mathrm{s}$ for a 3 -s gust with a maximum of $48 \mathrm{~m} / \mathrm{s}$. Those high values come from a time period with several storm events such as Storm Deirdre between 14 and 15th of December 2018 with large impact including local severe gales and heavy rain in the South West UK, including a wind gust of $79 \mathrm{mph}$ at the Welsh coast. Some other events were captured between the end of January and beginning of February 2019 as shown in Fig. 4. A wide range of low, mid and high wind speeds was also found, providing enough load cases to extract for the analysis, satisfying the purpose of the SHM by capturing a

Table 1 Selected site details

\begin{tabular}{llllll}
\hline Site name & Structure type & Height & Foundations & Top frame & Cable location \\
\hline St Ives FC & Portasilo & $17.5 \mathrm{~m}$ & Pad: $3.6 \times 3.6 \times 0.85 \mathrm{~m}$ & Mounting pole & External \\
\hline
\end{tabular}




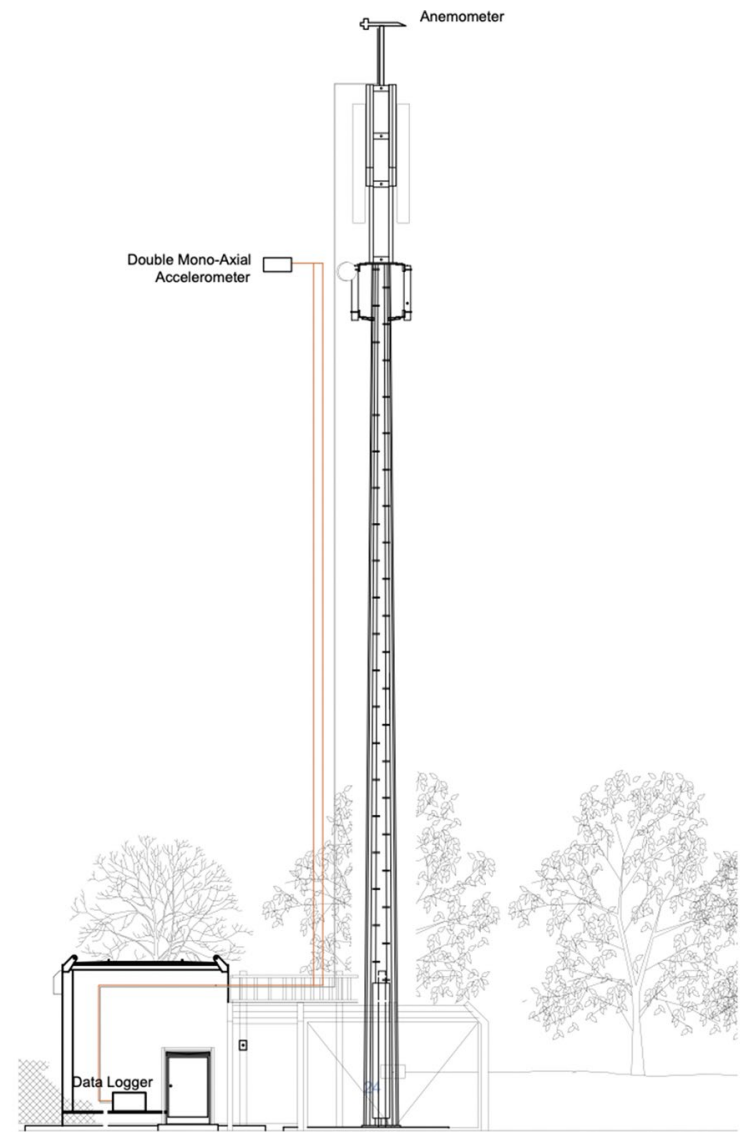

Fig. 3 Elevation and Plan View of Monitored Site. St Ives FC monopole

wide range of ambient excitation. The bottom plot in Fig. 4 shows the structural response recorded by the two accelerometers. There is a good correlation between accelerations and wind speeds, suggesting a clear along-wind response.

In terms of wind directions, the centre plot on Fig. 4 shows highest events for atypical wind directions in the UK where the strongest winds are predominant from the southwest quadrant. In this location, there are topography aspects which negate the existence of this main directions, having up-hill slope resulting in strongest winds from the northeast direction. To examine the nature of the turbulence in the incident wind it is conventional to plot gust wind speed as Fig. 4. Comparison of mean and peak values in this plot suggests that high intensity of turbulence is found for the range of mean wind speeds.

The results agree with expectations. The anemometer at $17 \mathrm{~m}$ is not high enough to avoid high turbulence of winds coming across rough terrain. Current formulations managing dynamic performance due to buffeting neglect turbulence and apply a gust factor, an approach that works properly for high structures like skyscrapers, high lattice towers or high guyed masts where wind tends to have relatively low turbulence. Monopoles operate in high turbulence environments which dominate the response behaviour.

Figure 5 shows the correlation between wind speed and response. This behaviour is typical for dynamic structural response to turbulent buffeting via along-wind excitation. Figure 5 shows no evidence of high amplitudes of response for wind speed ranges between 5 and $20 \mathrm{~m} / \mathrm{s}$, where vortex shedding [17] would appear. The structure responded only under along-wind turbulent buffeting loading.

The data provided by the SHM system were sufficient to characterise the full range of dynamic behaviour under wind loading. The next section will describe the application of several OMA methods, implemented in different software, to understand the evolution of dynamic modal parameters at different response levels and varying ambient excitation during the monitoring. Experiences from this exercise would be relevant for similar SHM systems on slender communications structures.

\section{Operational modal analysis}

The application of operational modal analysis (OMA) is attractive in practice because it avoids the logistical challenges of using mechanical shakers, using service windambient loading.

\subsection{Power spectral densities and their singular value decomposition}

The study of frequency domain metrics like power spectral densities (PSD) of the response is a common starting point for investigating modal properties via OMA. A frequency band with PSD exhibiting dynamic amplification is a reflection of resonance and hence potential modes, but it does not directly indicate the number of modes. The latter can be investigated via the singular value spectrum, i.e., a plot of singular values of the cross-spectral density matrix. Within a frequency band, the number of lines taking the shape of dynamic amplification indicates the dimension of the subspace spanned by the mode shapes, which is often equal to the number of modes in the band.

The structural response of a monopole under normal buffeting is defined by an oval/elliptical horizontal movement with major axis in the along-wind direction. The circular geometry of the shaft along its height, and the asymmetries found on the structure like the ladders, internal and external feeder cabling and lump-mass ancillaries result in a pair of related modes with close frequencies. These correspond to the main cantilever flexural bending modes as shown on Fig. 6 extracted from St Ives monitoring. The second and third modes expected between 3 and $6 \mathrm{~Hz}$ do not appear clearly on the singular value spectrum; they might be excited 
Fig. 4 Monitoring data in $\mathrm{St}$ Ives FC. Top. Wind speed values (Raw, mean and gust). Centre: Wind direction (Degrees). Bottom: Response captured (Accelerations)
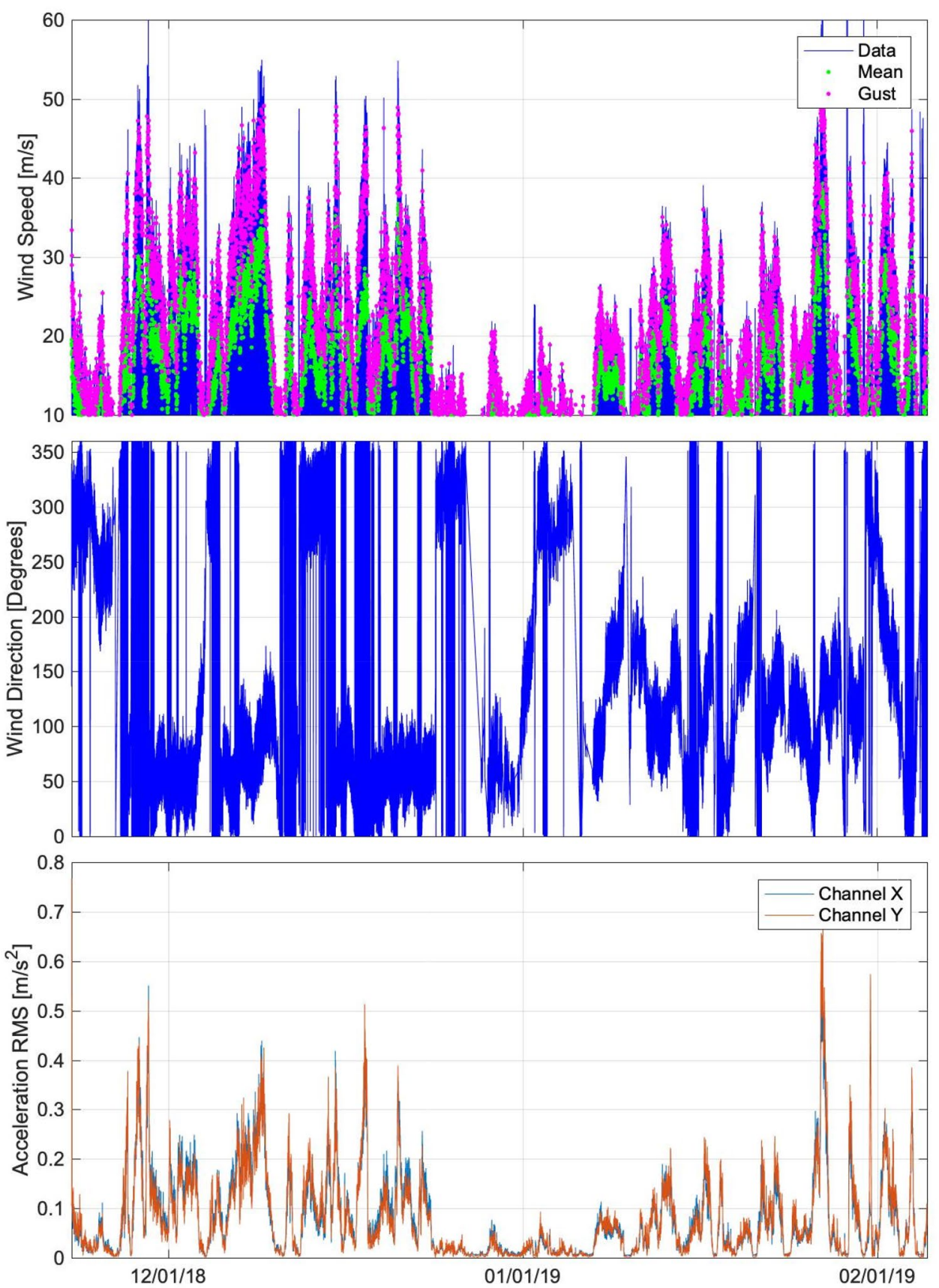

under aeroelastic effects or extreme wind event. Some 'fake' peaks are found due to analogue of transients (spikes, shifts) from $\mathrm{AC}$ equipment which are highly dissipated with high responses.

Bayesian OMA method (BAOYMA) has been implemented to track the modal parameters under ambient loading and yield the 'most probable' modal parameters and 'posterior' (i.e., given data) variance that measures the identification uncertainty. The posterior variance or uncertainty bound informs about the identification uncertainty given a particular set of data but not the variability of modal parameters over the duration of the time frame. BAYOMA needs to be investigated in an ensemble manner based on the identification results of different data sets.

The main purpose of this kind of monitoring is to provide more knowledge about the ambient loading related to wind in transient and rapid changes, analysing likely high response under buffeting and unlikely high response under vortex shedding. When there is clear time-variation of response signals due to changing modal properties, with impact on stationarity requirements, the $T_{\mathrm{fr}}$ of the evaluation window for OMA should trade a balance 


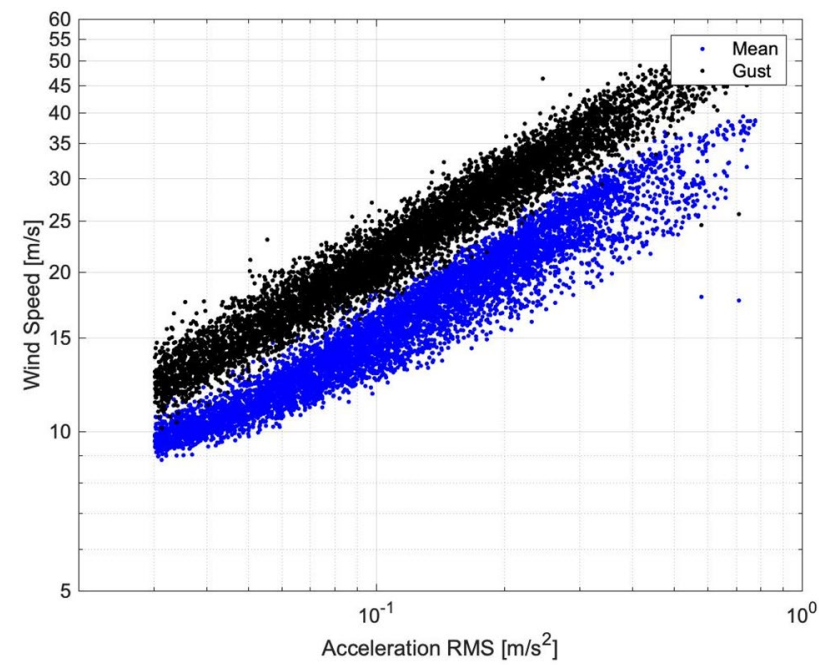

Fig. 5 Diagram of relationship between RMS and wind speed

between identification uncertainty (the longer the better) and modelling error especially with regard to stationarity (the shorter the better). $T_{\mathrm{fr}}$ of $10 \mathrm{~min}$ was found adequate to capture environmental wind variations while obtaining modal estimates with acceptable quality, and it is a conventional value for mean wind speeds in some codes.

\subsection{Bayesian OMA}

BAYOMA $[10,18]$ works in the frequency domain through the Fast Fourier Transform (FFT) of ambient vibration time series on a selected frequency band covering the modes of interest. The FFT in the selected band is used directly without windowing or averaging. The FFTs outside the selected band are not used and hence do not affect identification results. Given information from data and modelling assumptions, the uncertain modal parameters have approximately a joint Gaussian distribution. The mean of the Gaussian distribution is the most probable value (MPV), which minimises the negative log of likelihood function (NLLF). The covariance matrix can be calculated as the inverse of Hessian of NLLF at the MPV. Efficient algorithms and computer codes have been developed for the computation of MPV and covariance matrix. Each diagonal entry of the covariance matrix gives the variance $\left(\sigma^{2}\right)$ of the corresponding parameter. In this study, the coefficient of variation (c.o.v.), i.e., ratio of standard deviation $\sigma$ to the MPV, will be reported as a dimensionless measure of identification uncertainty.

BAYOMA involves solving an optimisation problem to obtain the MPV and the algorithm is iterative in nature. It is often more time consuming than explicit identification methods like SSI, especially for close modes. Well-separated modes can typically be handled in a matter of seconds while the computational time for close modes ranges from a few seconds to a few minutes, depending on factors such as the
Fig. 6 Power Spectral density and Singular value (SV) spectrum of ambient response data spectra, with corresponding mode frequencies identified by operational modal analysis (OMA) of initial assessments. Error bars indicate frequency bands manually chosen for BAYOMA identification and dots indicate extracted mode frequency (most probable values, MPV)
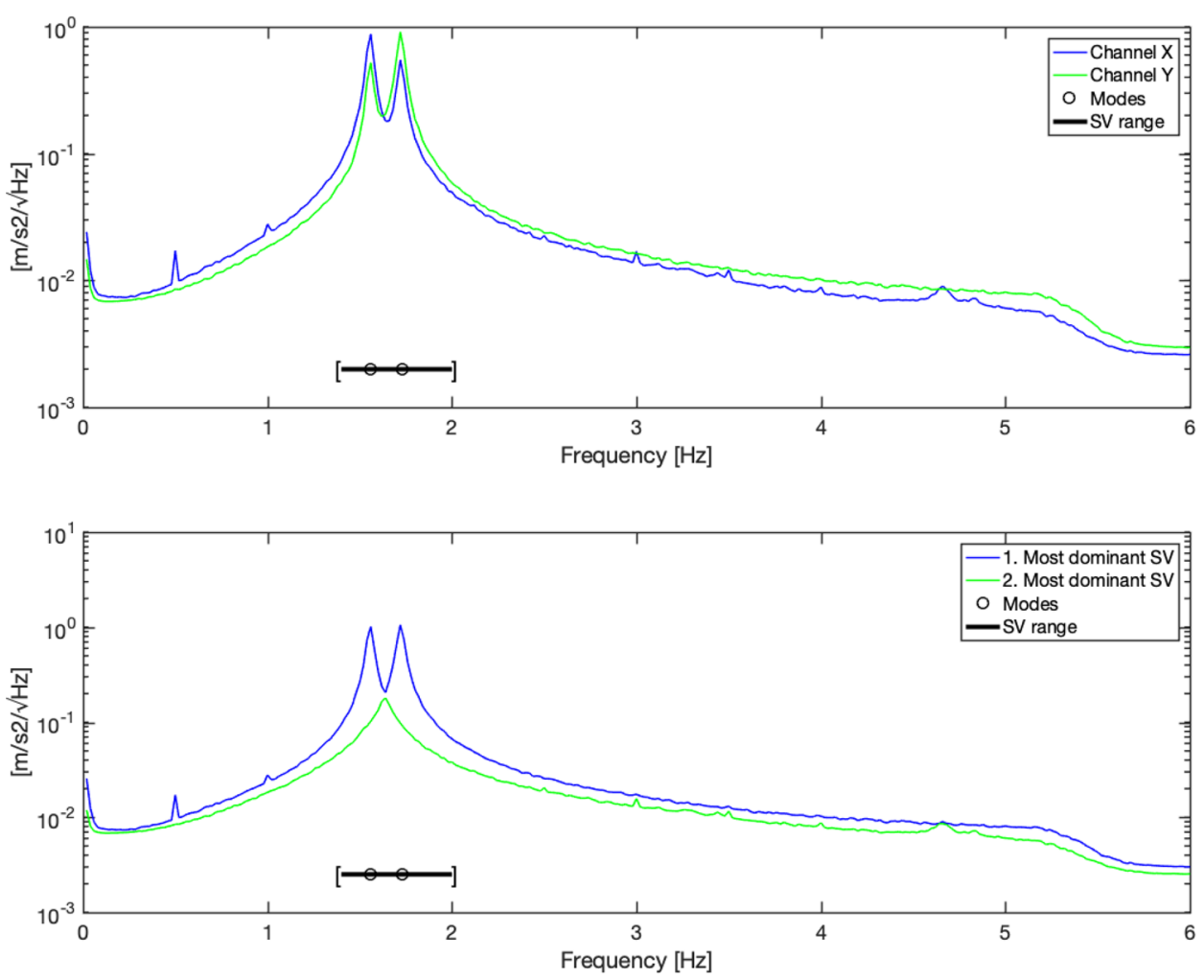
number of modes (the more the longer) and potential modelling error in data (the higher the longer). Recent developments in Ref. [19] provide analytical formulas for assessing the identification uncertainty of modal parameters in terms of test configurations, which is of practical relevance in ambient vibration test planning. Some applications of BAYOMA are presented in Refs. [20-22].

As indicated in Fig. 6, the initial values for MPV of natural frequencies for the first two modes are taken (handpicked from figure) to be $1.556 \mathrm{~Hz}$ and $1.723 \mathrm{~Hz}$. The frequency band whose FFT will be used for identifying the modes are taken to be 1.4-2 Hz.

As for other OMA methods that assume time-invariant and stationary models, applying BAYOMA to a single time window does not directly allow one to quantify the variation of MPs over an extended duration. Currently a simple empirical way to track variation is to apply them to different $T_{\mathrm{fr}}$ and track the results accordingly.

\subsection{Time variation and correlations in modal parameters using BAYOMA}

The coloured and possibly time-varying nature of wind speed or human forcing (e.g., due to climbing the mast) changes the spectrum of ambient dynamic loading. Other ambient factors such as temperature and humidity can lead to variation of MPs and collectively result in non-stationary response. The diagnosis of structural issues identified via changes in MPs, and model updating have been a particular problem under investigation in structural dynamics. To implement the method over the whole set of data, the $T_{\mathrm{fr}}$ is a key input that affects the estimation of MPs and the relationships among load and response parameters.

To study the MPs behaviour under wind-ambient loading, one requires data that are strongly non-stationary over the duration while being sufficiently stationary within $T_{\text {fr }}$. Long-term monitoring is one option to meet both requirements. In the present study, storms with high gust wind speed and buffeting response have been captured by the monitoring data.

Figure 7 shows the response of St Ives from 9 to 10th of December of 2018, during passage of Storm Deirdre for three different values of $T_{\text {fr }}$ In first row plot, responses follow wind speed, as was confirmed before on Fig. 5. Second, third and fourth row plots show estimations of MPs (Natural frequency, modal damping and mode shape orientation) for both modes with error bars representing $\pm 1 \sigma$ identification uncertainty about MPVs. Both vertical axes are utilised to visualise properly the variation of frequency and mode shape direction for both modes. Using the same frequency range
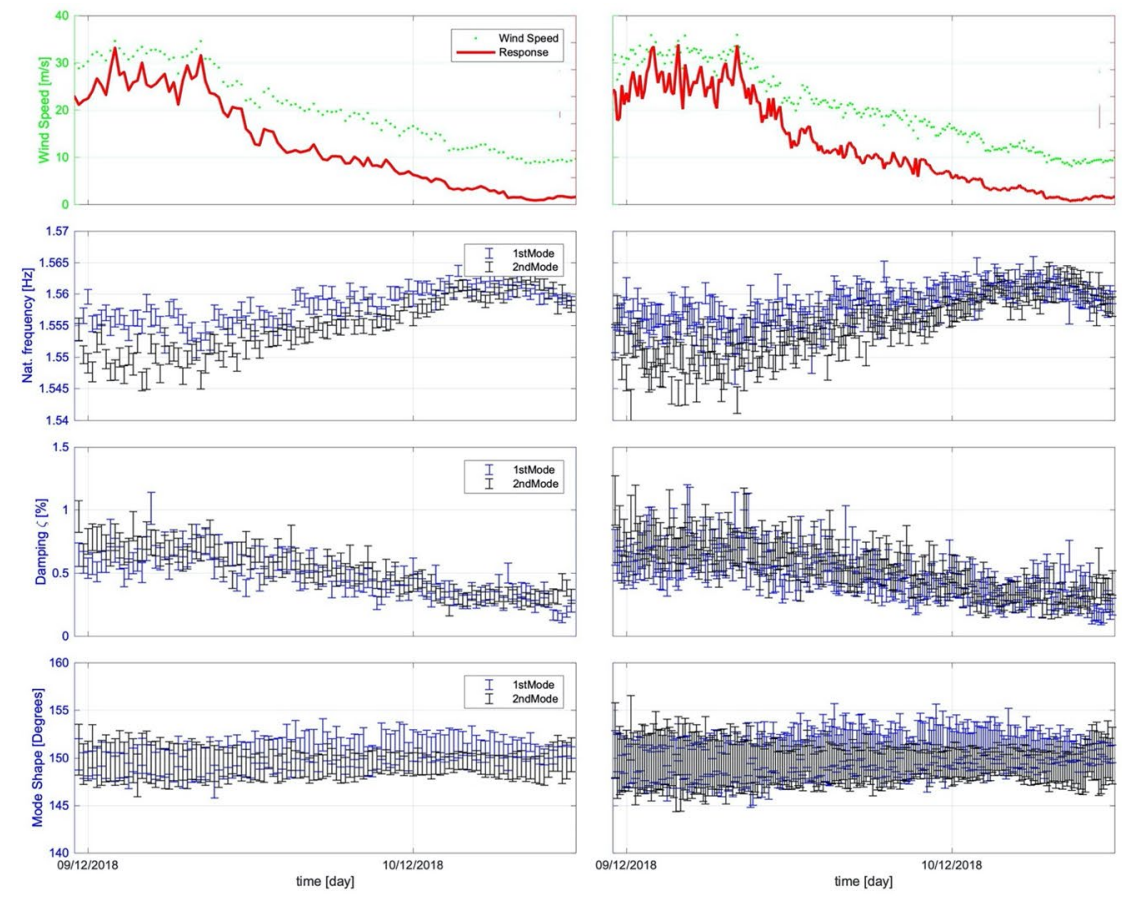

mode in blue (left $y$-axis) and second mode in black (right $y$-axis). Size of error bar reflects $1 \sigma$ identification uncertainty on individual estimate. Right columns: $T_{\mathrm{fr}}=5 \mathrm{~min}$. Centre columns: $T_{\mathrm{fr}}=10 \mathrm{~min}$. Left columns: $T_{\mathrm{fr}}=20 \mathrm{~min}$
Fig. 7 Wind speed and response obtained in St Ives FC during Storm Deirdre, 9-10 Dec 2018-first row. Application of OMA: BAYOMA on response data St Ives FC-Top to bottom: Frequency, damping and mode shape variation (reference angle from accelerometer-Channel X, anticlockwise positive) for first and second mode shape. First
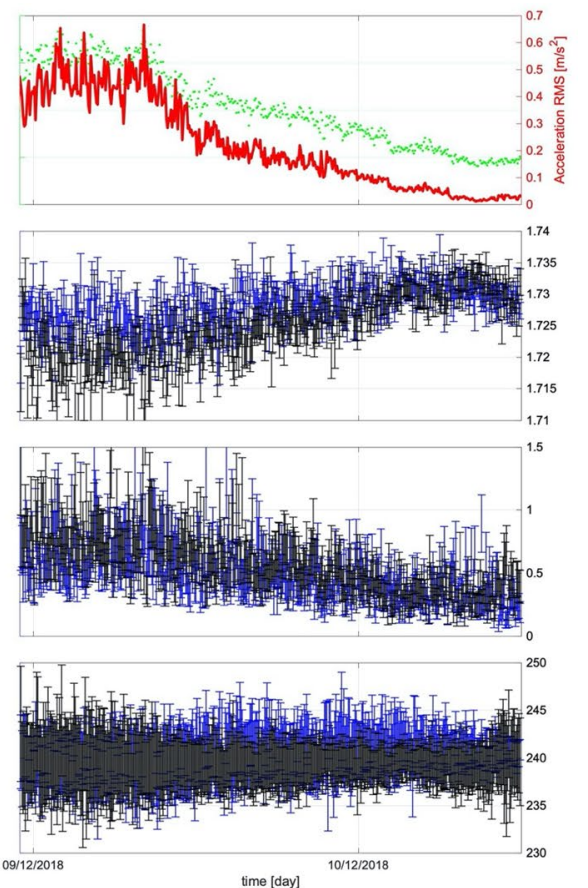

time [day (1) 
among the plots, highlights the differing 'closeness' as well as the inherent MP variation.

The centre column shows also estimations of MPs for $T_{\mathrm{fr}}=10$ min segment. Slow decrements of frequency are found in both modes for rising response levels. Damping exhibits higher values due to either amplitude dependency of structural damping or the aerodynamic damping contributions. However, the difference between mode direction changes little (the angles shown in the bottom plot track each other) showing much higher dependency of the geometry factors rather than possible ambient loading characteristics.

Figure 7 also presents BAYOMA analyses applied to data during the same selected period using time segments of $T_{\mathrm{fr}}=20$ (left column) and $T_{\mathrm{fr}}=5 \mathrm{~min}$ (right column). A coefficient of variation, c.o.v. $<0.2$ for damping estimates could be considered as acceptable criteria: $T_{\mathrm{fr}}=5$-min provides c.o.v. $>0.2$, while for $T_{\mathrm{fr}}=20$-min keeps c.o.v. $<0.1$. The effect of changing $T_{\text {fr }}$ can be seen in the error bounds in the plots. The same slow variations in MPV (with similar clear and consistent trends) are shown for the three segment lengths and clearly the longer segments have reduced variance (which goes approximately with square root of data duration). There appears to be no greater uncertainty where the frequencies (or rather their MPVs) are changing faster with time.

The identification uncertainty associated with an MP estimate from longer duration data $T_{\mathrm{fr}}=20 \mathrm{~min}$ of merged data would not reveal such variation since it assumes time-invariance over the duration. Similarly, variation of each mode shape MPV in polar coordinates is improved with longer data. One motivation to monitor a structure is to identify factors (such as load variation) that govern the dynamic behaviour in terms of MPs. In this case, shorter $T_{\mathrm{fr}}$ can capture the effect of faster changes in loading and so the selection of $T_{\mathrm{fr}}$ becomes an important issue. Based on these concerns, $T_{\mathrm{fr}}=10 \mathrm{~min}$ is found to agree with good correlated damping, while being able to achieve high percentages of the peak responses of the system. Using $T_{\mathrm{fr}}=10 \mathrm{~min}$ is also consistent with wind engineering practice where it is a standard averaging time for wind speed statistic used in structural engineering.

\subsection{Frequency and damping estimation}

The correlations between response data, wind loading and variation of MPs (stiffness and damping) can be seen in Figs. 8, 9.

In terms of frequency, the trend of both modes shows an amplitude dependency behaviour at high levels of excitation. The restoring forces will exhibit a nonlinear behaviour which might be classified as a softening characteristic [23]. Similar Portasilo monopoles have experienced structural problems due to dynamic behaviour in the last decade, e.g.,
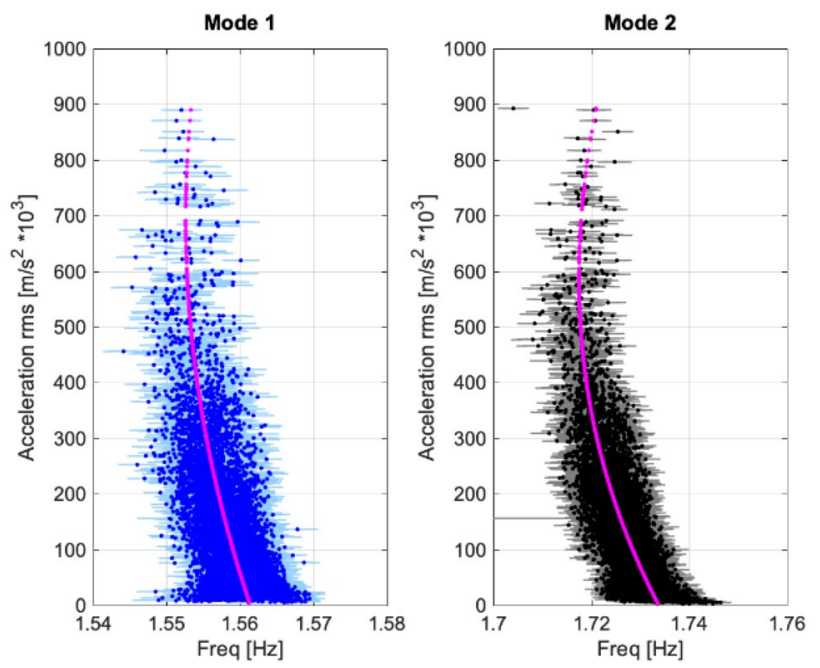

Fig. 8 Variation of Frequency with Response, BAYOMA
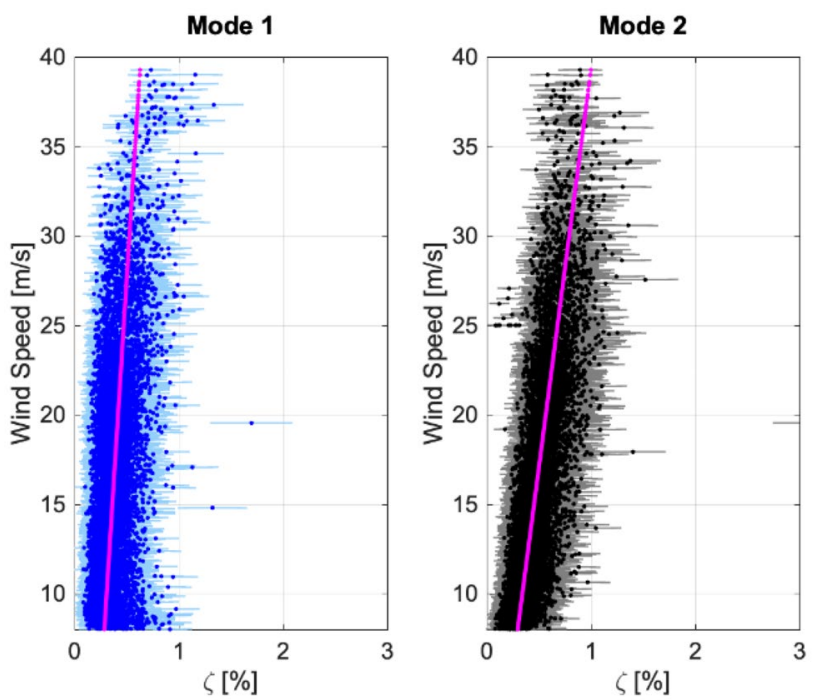

Fig. 9 Variation of Damping with Response, BAYOMA

due to fatigue failures at base inner welded connections. Figure 8 shows lower frequencies than calculated in analytical models during dynamic assessments, i.e., Portasilo monopoles tend to be more flexible than estimated by numerical models. This issue might involve a possible deterioration due to the inner connections, or wrong detailing provided by manufacturer which must be considered to modify and validate current models. Structural diagnosis can be carried out by comparison with other monitoring systems or existing previous data.

The difficulty of estimating damping is commonly recognised. Three main origins of damping include: (1) structural damping, appearing without external loading influence due to material, connections, construction method or foundation; 
(2) aerodynamic damping induced by the interaction of structure motion with wind; and (3) external damping provided by specific dampers. Currently, a common value of structural damping and a modal aeroelastic approach are given by different literature advising a linear increment of total damping with wind speed.

Figure 9 shows results reflecting the mentioned linear variation of total damping with response, although existing standards do not take into account the response based on two modes. Structural damping could be extracted from damping values under small excitations with minimum wind influence. At that level, BAYOMA provides high confidence in a constant term of $\zeta=0.4 \%$, onto which amplitude- and wind-dependent contributions would be added.

Figure 9 shows an expected linear aerodynamic component of damping related with levels of wind speeds as the Institution of Lighting Engineers (UK) Technical Report Number 7 [3]. However, existing methods of assessing aerodynamic damping do not agree with obtained values, suggesting values up to three times higher. This issue involves the redefinition of proposed formulation for damping or wind resistances including drag factors to develop in further investigations.

Both parameters show a disagreement in comparison with recommendations. Measurements show lower frequency, higher structural damping and lower aerodynamic damping which compromise current structural assessment with higher dynamic augmentation factor used among the quasistatic procedure suggested by the main Standards. In addition, elements such as the amplitude dependency of natural frequency or the existence of a secondary mode show the crude code approaches to be inappropriate.

\subsection{Mode directionality}

The identification of the mode shapes is a challenging issue in symmetric structures. For square or rectangular buildings modes tend to be aligned with structural axis of symmetry. However, previous experiences in flexible lattice towers show strong dependence on (mis-)alignment of the mass and structural features along the height of the structure.

For Monopoles with circular section in plan view, the predominant mode directions are theoretically arbitrary and in reality they are sensitive to asymmetries in stiffness, mass and boundary conditions. Monopoles maintain symmetry along the shaft of the monopole with necessary openings and steelwork introducing minor symmetry disruptions. Variation in steel and base grout stiffness, mechanical fixing between courses (by keying and bolts) or foundationsoils softness may not have significant effect compared to the necessary access steelworks such as ladder and anticlimbing elements that breaks the alignment adding stiffness to some specific directions. This misalignment is even more evident with mass distribution: heavy antennae at different levels and in different directions disturb the symmetry. In addition, feeder cables, normally run internally, can be externally installed and consequently promote a specific orientation for each mode. All these factors result in two close-frequency orthogonal main modes-directions, the lowest frequency mode with less stiffness aligning usually perpendicular to ladder direction.

As shown in Figs. 1, 7, St Ives Monopole has all the aforementioned ingredients. New monopoles specifically designed to be installed in cities avoid access requirements and shrouded elements which make them to appear totally symmetric. In those cases, hidden panels are not symmetric which induce the differences between modes. However, both modes will appear closer than any other types.

The St Ives FC mode shapes obtained by BAYOMA are presented in Fig. 10. The MPVs are illustrated as vectors which are consistent with orientation shown in Fig. 7. Being vector-valued the dominant identification uncertainty of each mode shape is obtained from the eigenvector (maximum eigenvalue) of its posterior covariance matrix multiplied by the square root of the eigenvalue. This $\pm 1 \sigma$ uncertainty is shown as (magenta) lines, which are roughly perpendicular to the vector tip and parallel to the coupled mode. The blue (and black) arrows show the most probable mode shape directions from different data sets. Their variability is an aggregate of identification uncertainty (in each data set) and possible variation in the actual mode shape directions (during different data sets). In the present case the variability of the blue arrows is roughly of the same order of magnitude as the identification uncertainty suggested by the magenta arrows. This suggests that the variability over different data sets is not significant. Both modes appear to have a narrow band with low uncertainty which might suggest a non-winddirectional dependency.

\section{Implementation of alternative operational modal analysis: SSI in ARTeMIS}

To provide a supplementary qualitative view of the identified modal properties (e.g., for counter-checking), Stochastic subspace identification (SSI) technique [24, 25] that is common in the literature was also implemented. SSI is based on a time-domain state-space model of successive data. The state matrices are estimated by means of regression, from which the MPs are back-calculated assuming structural dynamics. SSI is attractive in applications as the calculations are explicit, requiring no iterations and being immune from convergence issues. Operating in the timedomain, the unknown excitations and instrument noise are effectively assumed to have constant spectral characteristics from DC up to the Nyquist frequency. As modal properties 
Fig. 10 Main bending cantilevered modes orientation in plan view for St Ives monopole (top). Picture of external cabling and ladder installation (bottom)
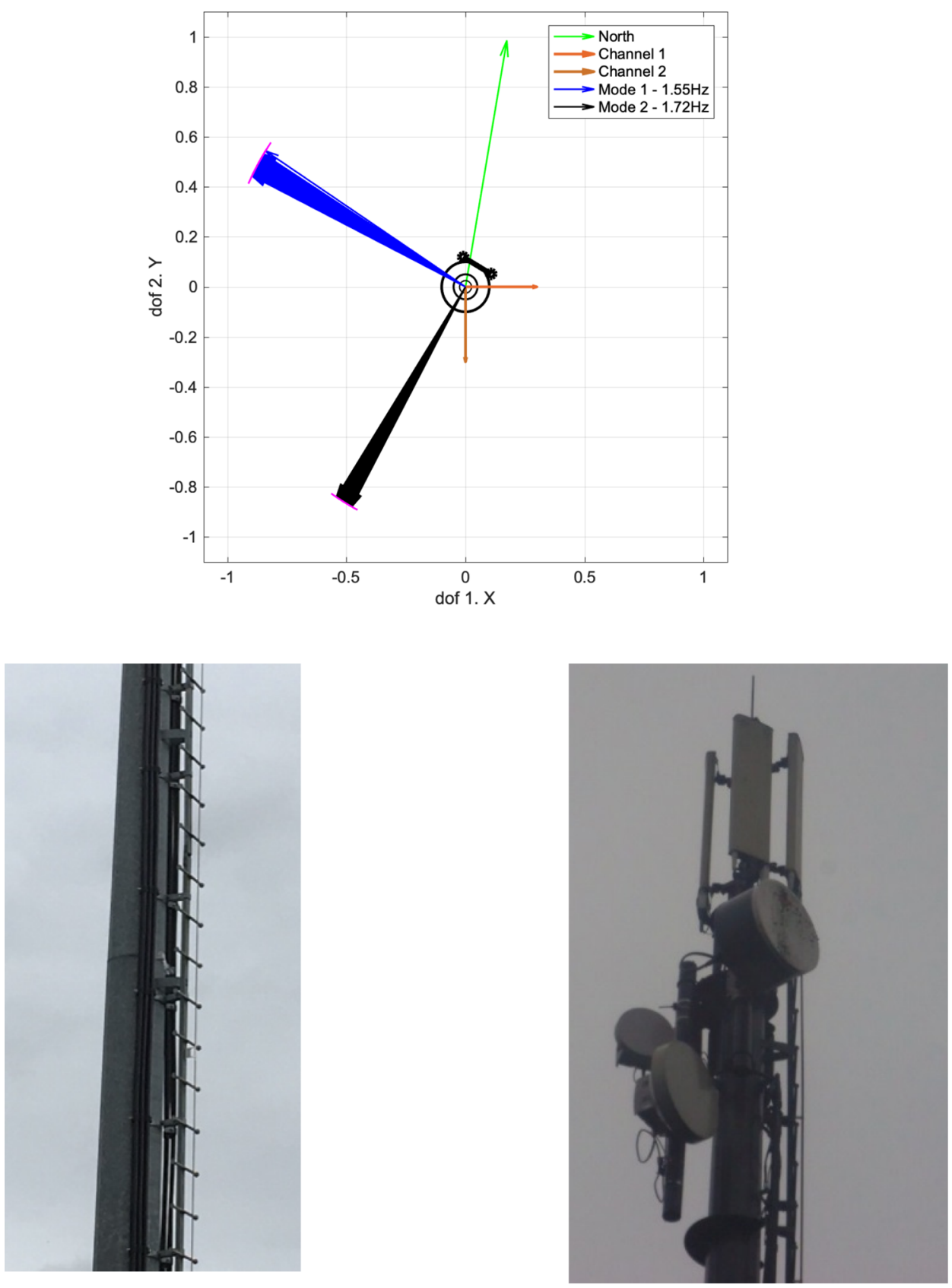

are back-calculated from estimated versions of state matrices they need not obey structural dynamics, however, which is a source of modelling error. Naturally, the effect on the identified modal parameters depends on how well the state matrices are estimated, which in turn depends on factors such as data length, signal/noise ratio or how well the data agree with structural dynamics.

In this study, SSI was executed on data resampled at $12.8 \mathrm{~Hz}$ with order up to 100 poles through ARTeMIS Software [15]. Three common techniques were used, including the unweighted principal component (SSI-UPC), principal component (SSI-PC) and canonical variate analysis
(SSI-CVA). Similar comparison exercises have been presented in Refs. [14, 26-30] describing two decades of application of OMA to investigate time-variation of MPs of important civil engineering structures.

As part of the SHM procedure, the collected response time series were processed using an OMA procedure that fits a state space model to the monopole structure responses driven by ambient excitation [31]. As mentioned from existing experiences, this exercise expects to obtain a non-complex power spectral density with a pair of close modes between 1 and $2 \mathrm{~Hz}$, and second and third pairs between 3 and $6 \mathrm{~Hz}$. Each setup leads 
to a stabilization diagram such as last plot of Fig. 11 . Finally, both most remarkable modes are clearly identified, $f_{1}=[1.54-1.58] \mathrm{Hz}$ and $f_{2}=[1.7-1.75] \mathrm{Hz}$. Results from SSI under low response are rejected and not taken into the analysis estimation (Fig. 11).

\subsection{Discussion between OMA identification systems}

The St Ives SHM system provided 76 days of synchronized wind and response data that are helpful to compare how current design static and dynamic approaches predict the behaviour of slender monopole structures. This set of data is probably the only one known on monopoles to authors at
Fig. 11 Acceleration response data (two top time series), Stabilization SSI-PC diagrams and PSD applied to selected data in St Ives FC obtained from ARTeMIS software
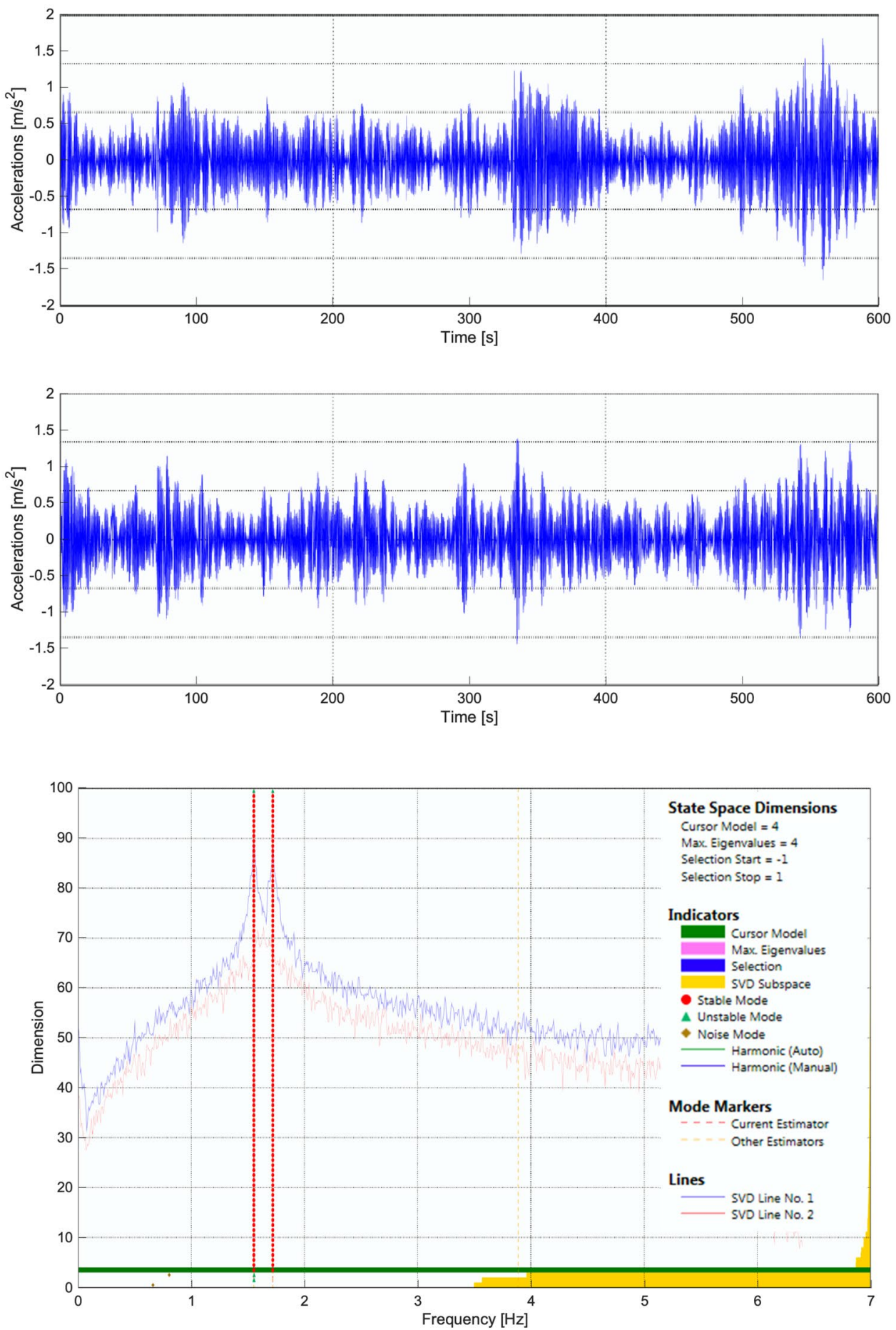
the present state of the art: such full-scale data with reliable OMA provides essential addition to current knowledge to support advances compared in code provision.

As well as improving the analytical model validation and operational model assessment, the exercise demonstrates the competences and restrictions of the selected techniques for analysing data from future permanent monitoring systems in similar communication structures. For comparison reasons, only high response segments above $\mathrm{rms} 0.580 \mathrm{~m} / \mathrm{s}^{2}$ are presented here. This results in 400 data segments of $10 \mathrm{~min}$ and correspondingly 400 sets of identification results. Each set of identification results includes, for each modal parameter, $\mu=$ posterior MPV (BAYOMA) or best estimate (SSI) and $\sigma=$ standard deviation (BAYOMA/SSI) that measures the identification uncertainty. Specific coding was built to link MATLAB and ARTeMIS making the analysis and the collection of the results more feasible.

Table 2 gives a representative picture of the identification results in terms of the average values of $\mu$ and $\sigma$ over the 400 sets of results. The results are plotted in Fig. 12 with respect to acceleration rms on the vertical axis. Implementationwise, BAYOMA requires initial guess of natural frequency and selection of frequency band covering the mode(s). SSI requires the choice of Hankel matrix size and order of state-space system. The average values of $\mu$ from SSI and BAYOMA are similar for both natural frequencies and damping ratios. The same is not true for $\sigma$, however, with values from SSI generally lower than those of BAYOMA. A formal analysis of this difference is out of the scope of this work, as the methods operate on different domains (time vs frequency) and are based on a different set of assumptions (e.g., state-space vs structural modal, white noise in time vs flat PSD on selected band) and effective data bandwidth used (from near DC to Nyquist in SSI vs the resonance band in BAYOMA). As a standard result in classical statistics, if there is no modelling error then the ensemble variability of any unbiased estimator is always greater than the CramerRao bound, which coincides with the variability of the maximum likelihood estimator (MLE). The MLE is numerically equal to the MPV of BAYOMA. Aside, recent work allows the value of $\sigma$ to be explained in terms of test configurations, in the context of BAYOMA [32].

\section{Conclusions}

Under the loading changes demanded by new mobile technologies, better knowledge of dynamics in communications structures is necessary. A Structural Health Monitoring system has been satisfactorily developed for telecoms monopoles with height ranging $15-30 \mathrm{~m}$. This allows the present study to analyse the wind excitation-response relationship, to provide better understanding of structural behaviour in wind-ambient loading and to investigate the evolution of modal parameters with different levels of excitation.

A large dataset of acceleration data from installation on a monopole in St Ives, Cornwall, has been analysed. Modal properties of the first few harmonics along two principal directions have been identified using a Bayesian OMA method (BAYOMA). In addition to the most probable value, the method also provides the identification uncertainty, which is important for downstream investigation of potential relationships. As is conventionally done, structural and statistical wind load properties are assumed to be constant in each short time window for analysis, where a duration of $10 \mathrm{~min}$ has been found to balance identification uncertainty (the longer the better) and modelling error (the shorter the better).

The performance of modal properties during a wide range of wind events and consequent response amplitude provide important features to study in further structural engineering valuations. Stiffness exhibits a softening nonlinear amplitude dependence behaviour on both main modes. Damping shows a clear linear wind-speed dependency, which advice aerodynamic contributions considerable lower than given by some national codes, shortest value in calm weather at $0.4 \%$ can be taken as structural damping value. Those discrepancies will confirm the need of further investigations to implement changes.
Table 2 Frequency and Damping estimation extracted from ARTeMIS-SSI and BAYOMA identification method

\begin{tabular}{|c|c|c|c|c|c|c|c|c|}
\hline \multirow{2}{*}{$\begin{array}{l}\text { OMA } \\
\text { Techniques }\end{array}$} & \multicolumn{4}{|l|}{ Mode 1} & \multicolumn{4}{|l|}{ Mode 2} \\
\hline & $\mu(f) / \mathrm{Hz}$ & $\sigma(f) / \mathrm{Hz}$ & $\mu(\zeta) / \%$ & $\sigma(\zeta) / \%$ & $\mu(f) / \mathrm{Hz}$ & $\sigma(f) / \mathrm{Hz}$ & $\mu(\zeta) / \%$ & $\sigma(\zeta) / \%$ \\
\hline SSI-PC & 1.5543 & 0.0002 & 0.9167 & 0.0127 & 1.7194 & 0.0002 & 0.8090 & 0.0138 \\
\hline SSI-CVA & 1.5538 & 0.0015 & 0.9130 & 0.0791 & 1.7194 & 0.0010 & 0.7677 & 0.0649 \\
\hline SSI-UPC & 1.5538 & 0.0001 & 0.9271 & 0.0085 & 1.7195 & 0.0002 & 0.8101 & 0.0131 \\
\hline BAYOMA & 1.5532 & 0.0021 & 0.7423 & 0.1488 & 1.7185 & 0.0023 & 0.7972 & 0.1677 \\
\hline
\end{tabular}

The value of $\mu$ (posterior MPV or best estimate) and $\sigma$ (identification uncertainty) presented here are average values over identification results of 400 data samples above RMS $0.580 \mathrm{~m} / \mathrm{s}^{2}$ to give a representative measure. See Sect. 5.1 for discussion 
Fig. 12 Modal Parameters estimation above RMS $0.580 \mathrm{~m} /$ $\mathrm{s}^{2}$ using ARTeMIS-SSI and BAYOMA
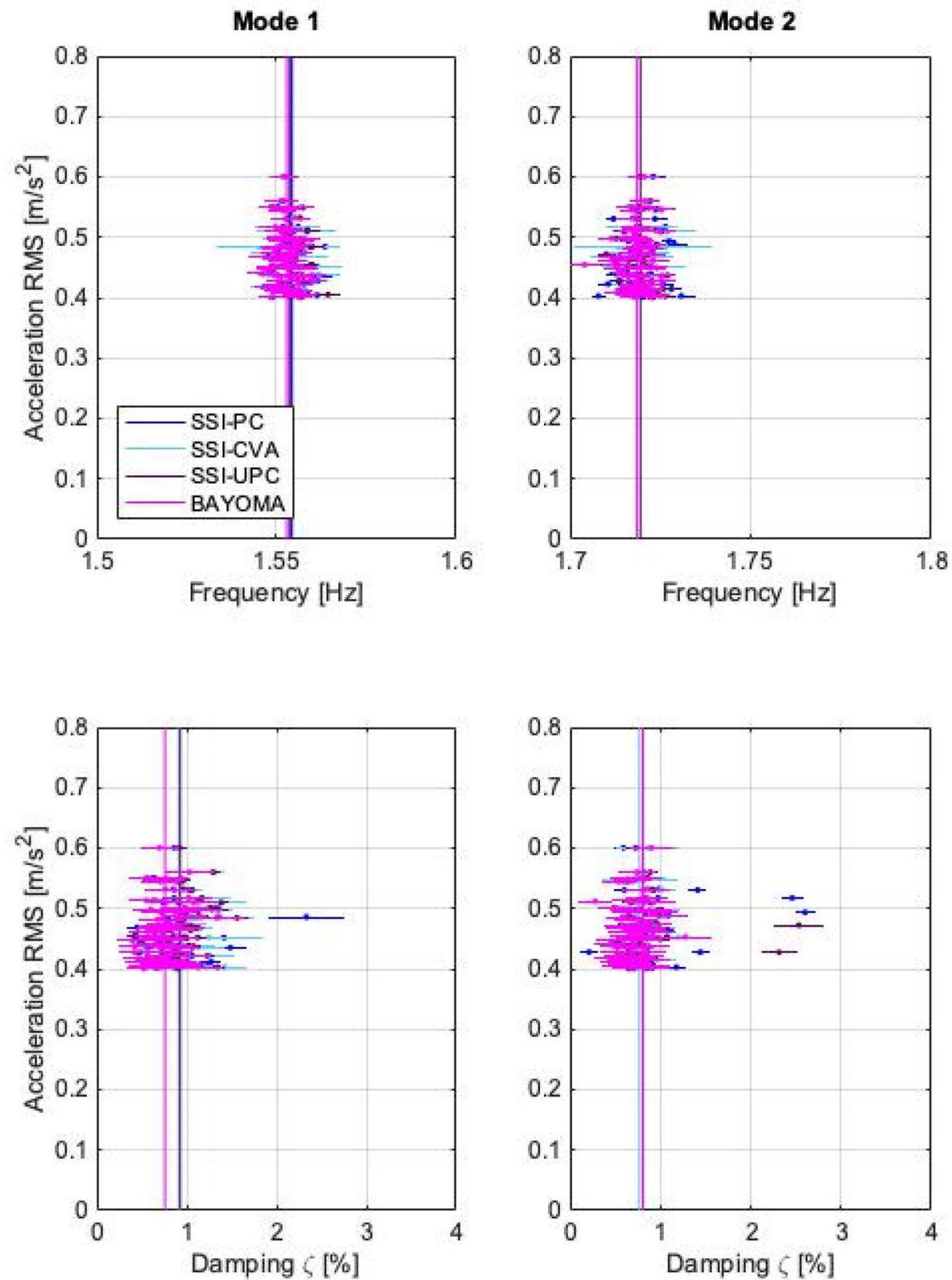

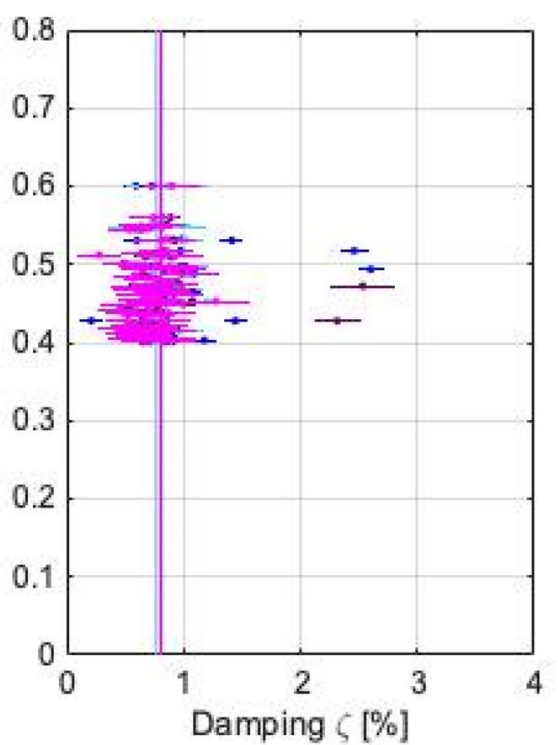

Consistent with physical intuitions, the fundamental first mode shape directions for monopoles are predominantly aligned according to external elements such as access ladder or cabling. Symmetric geometry does not provide significant evidences, although, external elements defines a specific orientation for each mode of the main cantilevered bending mode shapes.

The monitoring results obtained under BAYOMA, and compared to stochastic subspace identification on standardised software, present opportunities for further studies in terms of understanding dynamic response in monopoles involving codes reformulation, structural diagnosis and model validation.

Acknowledgements The authors would like to thank the reviewers for their helpful comments. Support for this project was provided by Vibration Engineering Section of University of Exeter and Arqiva Ltd as owner of the structures tested and sponsor of the PhD Research.

Open Access This article is licensed under a Creative Commons Attribution 4.0 International License, which permits use, sharing, adaptation, distribution and reproduction in any medium or format, as long as you give appropriate credit to the original author(s) and the source, provide a link to the Creative Commons licence, and indicate if changes were made. The images or other third party material in this article are 
included in the article's Creative Commons licence, unless indicated otherwise in a credit line to the material. If material is not included in the article's Creative Commons licence and your intended use is not permitted by statutory regulation or exceeds the permitted use, you will need to obtain permission directly from the copyright holder. To view a copy of this licence, visit http://creativecommons.org/licenses/by/4.0/.

\section{References}

1. E. Commission (1991) BS EN 1991-1-4:2005 General actionsWind actions. Brussels

2. British Standards Institution (1986) BS 8100-1. Lattice towers and masts-Part 1: Code of practice for loading, vol 3, no 1. London

3. The Institution of Lighting Engineers (2013) PLG07: High Masts for lighting and CCTV

4. Lum E, Fatt P, Eng P, Jeyananda E, Ahnantakrishnan C (2017) Dynamic analysis of telecommunication monopole under wind loads, JURUTERA. Inst Eng Malaysia, pp 26-29

5. Peil U, Nölle H (1992) Guyed masts under wind load. J Wind Eng Ind Aerodyn 43(1-3):2129-2140

6. Ogle MH, Smith BW, Lambert MJ (1988) Investigation of windinduced fatigue in tall guyed steel masts. J Wind Eng Ind Aerodyn 30:55-65

7. Glanville MJ, Kwok KCS, Denoon RO (1996) Full-scale damping measurements of structures in Australia. J Wind Eng Ind Aerodyn 59:349-364

8. Glanville MJ, Kwok KCS (1995) Dynamic characteristics and wind induced response of a steel frame tower. J Wind Eng Ind Aerodyn 5455:133-149

9. Koo KY, Capilla JAJ, Brownjohn JMW (2019) Innovative sensing solution and SHM for Moel-Y-Parc telecommunication high guyed mast. In: International conference on smart infrastructure and construction 2019 (ICSIC), pp 589-594

10. Au SK (2012) Fast Bayesian ambient modal identification in the frequency domain, Part I: posterior most probable value. Mech Syst Signal Process 26:60-75

11. Au SK (2012) Fast Bayesian ambient modal identification in the frequency domain, Part II: posterior uncertainty. Mech Syst Signal Process 26:76-90

12. Brownjohn JMW, Au SK, Li B, Bassitt J (2017) Optimised ambient vibration testing of long span bridges. In: EURODYN 2017, p 10

13. Van Overschee P, De Moor B, Dehandschutter W, Swevers J (1997) A subspace algorithm for the identification of discrete time frequency domain power spectra. Automatica 33(12):2147-2157

14. Brownjohn JMW, Magalhaes F, Caetano E, Cunha A (2010) Ambient vibration re-testing and operational modal analysis of the Humber Bridge. Eng Struct 32(8):2003-2018

15. Extractor-ARTeMIS (1999) Structural vibration solutions. Aalborg

16. ESDU (2007) ESDU 77032: Fluctuating loads and dynamic response of bodies and structures in fluid flows-background information, pp 1-22
17. Belver AV, Koo KY, Ibán AL, Brownjohn JMW, Goddard C (2014) Enhanced vortex shedding in a 183 m industrial chimney. Adv Struct Eng

18. Au SK (2017) Operational modal analysis. Springer Nature, Singapore

19. Au SK, Brownjohn JMW, Mottershead JE (2018) Quantifying and managing uncertainty in operational modal analysis. Mech Syst Signal Process 102:139-157

20. Liu P, Lian PY, Yang WG (2018) Horizontal resonance of a 13 story building under external machine vibrations. Int J Struct Stab Dyn 18(1):1850005

21. Pan H, Xie Z, Xu A, Zhang L (2017) Wind effects on Shenzhen Zhuoyue Century Center: field measurement and wind tunnel test. Struct Des Tall Spec Build 26(13):e1376

22. Lam HF, Zhang FL, Ni YC, Hu J (2017) Operational modal identification of a boat-shaped building by a Bayesian approach. Eng Struct 138:381-393

23. Worden K (2019) Nonlinearity in structural dynamics: detection, identification and modelling. CRC Press

24. Peeters B, De Roeck G (2001) Stochastic system identification for operational modal analysis: a review. J Dyn Syst Meas Control 123(4):659-667

25. Mellinger P, Döhler M, Mevel L (2016) Variance estimation of modal parameters from output-only and input/output subspacebased system identification. J Sound Vib 379:1-27

26. Magalhães F, Caetano E, Cunha Á (2007) Challenges in the application of stochastic modal identification methods to a cable-stayed bridge. J Bridg Eng 12(6):746-754

27. Magalhães F, Caetano E, Cunha Á, Flamand O, Grillaud G (2012) Ambient and free vibration tests of the Millau Viaduct: evaluation of alternative processing strategies. Eng Struct

28. Diaferio M, Foti D, Mongelli M, Giannoccaro NI, Andersen P (2011) Operational modal analysis of a historic tower in Bari. Conf Proc Soc Exp Mech Ser 4:335-342

29. Tuhta S, Günday F (2019) Application of Oma on the bench scale aluminum bridge using micro tremor data. İnt J Adv Res Innovative İdeas Educ 5(5):912-923

30. Foti D, Ivorra S, Bru D, Dimaggio G (2012) Dynamic identification of a pedestrian bridge using operational modal analysis. In: Proceedings eleventh international conference on computational structures technology, pp 4-7

31. Van Overschee P, De Moor BL (2012) Subspace identification for linear systems: Theory-Implementation-Applications. Springer Science \& Business Media

32. Au SK, Brownjohn JM, Li B, Raby A (2021) Understanding and managing identification uncertainty of close modes in operational modal analysis. Mech Syst Signal Process 147:107018

Publisher's Note Springer Nature remains neutral with regard to jurisdictional claims in published maps and institutional affiliations. 